diagnostic yield for cardiac disease. Exclusion of these ECG parameters from the Group 2 category in asymptomatic athletes would reduce the false positive rate from $10 \%$ to an acceptable $<3 \%$.

\section{SEX AND ETHNICITY SPECIFIC ECG DIFFERENCES IN ELITE ATHLETES: RELEVANCE TO PRE-PARTICIPATION CARDIOVASCULAR EVALUATION: THE BRITISH EXPERIENCE}

doi:10.1136/heartjnl-2012-301877b.63

${ }^{1} S$ Gati, ${ }^{*}$ S Ghani, ${ }^{1} \mathrm{~N}$ Sheikh, ${ }^{1} \mathrm{~A}$ Zaidi, ${ }^{1} \mathrm{M}$ Papadakis, ${ }^{2} \mathrm{~L}$ Chen, ${ }^{1} \mathrm{M}$ Reed, ${ }^{1} \mathrm{~S}$ Sharma. ${ }^{1}$ St. George's University of London, London, UK; ${ }^{2}$ University Hospital Lewisham, London, UK

Purpose The athlete's ECG is affected by several demographic factors but there is a paucity of data relating to the impact of the athlete's sex and ethnicity. The ESC guidelines for ECG interpretation in athletes are derived predominantly from male cohorts. Extrapolating such criteria to athletes of African/afro-Caribbean origin and female athletes may lead to erroneous interpretation.

Methods Between 2001 and 2011, 1378 highly trained athletes (55\% males, $81 \%$ Caucasian) (mean age $21.6 \pm 5.43$ years); range 14-35 years, underwent cardiac evaluation including 12-ECG and echocardiography. ECGs were analysed for training related (group 1) and training-unrelated (group 2) changes, according to the ESC guidelines.

Results Males demonstrated a higher prevalence of Group 1 (89\% vs $61 \% ; p=<0.0001$ ) and Group 2 ECG changes (26\% vs $16 \%$; $\mathrm{p}=0.0001)$ compared with females. Of the group 1 changes, isolated left ventricular hypertrophy (42\%), early repolarisation patterns (ST elevation $>0.1 \mathrm{mV})(61 \%)$, first-degree AV block (10\%) were more prevalent in males compared to $14 \%, 45 \%$ and $4.7 \%$ females respectively $(p=0.0001)$. Of the group 2 changes, $T$-wave inversion in leads V1-V4 were more prevalent in female athletes $(12 \%)$ particularly black females $(17 \%)$ compared to male athletes $(4 \%$; $\mathrm{p}=0.0001)$, whereas, $\mathrm{T}$-wave inversion in the inferior leads were more common in males (3.3\% vs $0.6 \%$ ) irrespective of ethnicity. Males demonstrated a higher prevalence of axis deviation $(6.7 \%$ vs $2.1 \% ; \mathrm{p}=0.0001)$, atrial enlargement $(4.2 \%$ vs $1.0 \% ; \mathrm{p}=0.0002)$ and right ventricular hypertrophy $(\mathrm{RVH})(8.3 \%$ vs $2.6 \% ; \mathrm{p}=0.0001)$ compared with females. Caucasian athletes exhibited greater group 1 changes compared with black athletes $(73 \%$ vs $65 \%$; $=0.0161)$. Black athletes exhibited a higher prevalence of group 2 ECG changes compared with Caucasian athletes ( $34 \%$ vs $21 \%$; $p=<0.0001$ ) with $15 \%$ of black athletes exhibiting T-wave inversion, $4.5 \%$ left atrial enlargement $14 \%$ right atrial enlargement and $12 \%$ demonstrating RVH compared to $9 \%, 0.9 \%, 0.09 \%$ and $4.1 \%$ of Caucasian athletes respectively. There was no correlation between any ECG parameter and cardiac chamber size.

Conclusions Male sex and black ethnicity equated to a higher prevalence of Group 1 and 2 ECG changes compared with female sex and Caucasian ethnicity. However, anterior T wave inversion was significantly more common in females, being present in over $10 \%$ of athletes irrespective of ethnicity than previously reported. The precise incidence and significance of anterior $\mathrm{T}$-wave inversion in female athletes requires further assessment.

\section{PREVALENCE AND MORPHOLOGICAL CHARACTERISATION OF EARLY REPOLARISATION PATTERNS IN YOUNG HEALTHY INDIVIDUALS: IMPACT OF GENDER, ETHNICITY AND PHYSICAL ACTIVITY}

doi:10.1136/heartjnl-2012-301877b.64

S Ghani, ${ }^{*}$ S Di Fino, A Gravina, A Zaidi, N Sheikh, H Raju, M Muggenthaler, S Sharma. St George's University London, London, UK

Introduction Early repolarisation (ER) is commonly observed in athletes and young healthy individuals. Recently, ER in the inferior and lateral leads has been associated with sudden cardiac arrest from idiopathic ventricular fibrillation. We studied the prevalence, distribution and morphology of ER patterns in inferior and lateral leads in young healthy individuals.

Methods 12-leads ECG was performed at rest in 1237 young healthy individuals (age range 13-38 years) between February 2011 and September 2011. We evaluated the impact of gender, ethnicity and physical activity on ER. Individuals were divided into physicallyactive (exercise $>2 \mathrm{~h}$ /week) and sedentary. Early repolarisation was defined as notched or slurred J-point elevation of at least $0.1 \mathrm{mV}$ from baseline, in ¡Ý2 contiguous inferior or lateral leads; anterior ER patterns were not considered in this study. The morphology of STsegment was classified as horizontal/descending or rapidly ascending/up sloping.

Results The mean age of participants was 18.2 ¡ $\AA$ A 4.3 years, of which 979 (79\%) were male, 981 (79\%) were physically active and 91\% were Caucasians. ER pattern was present in a total of 232 $(18.7 \%)$ cases; of these $42 \%$ were in the inferior leads, $31 \%$ in lateral leads and $27 \%$ in both. Notched ER was more prevalent $(64 \%$ inferior, $83 \%$ lateral, $76 \%$ infero-lateral) compared to slurred morphology, and more commonly associated with ascending/ upsloping ST-segment elevation. ER was significantly more prevalent in males compared to females ( $20 \%$ vs $12 \%, p=0.003$ ), in physically-active people compared to sedentary $(20 \%$ vs $13 \%$, $\mathrm{p}=0.0194$ ), and in Afro-Caribbeans compared to Caucasians ( $40 \%$ vs $17 \%, p=0.0013)$. In addition, voltage criteria for left ventricular hypertrophy and sinus bradycardia were a common associated finding in individuals with ER pattern compared with those without ( $p=0.0001$ and 0.002 respectively). Only $5 \%$ of individuals with ER had J-point elevation of $>0.2 \mathrm{mV}$.

Conclusion Early repolarisation is a common finding in young healthy individuals, and is more prevalent in males, physicallyactive individuals and those with Afro-Caribbean ethnicity. Notched ER with ascending ST-segment elevation in inferior leads was the most commonly observed morphological pattern. More research is required to understand precise long-term implications of such repolarisation changes in young individuals.

\section{ATRIAL FIBRILLATION ABLATION IN A DISTRICT GENERAL HOSPITAL: 3 YEARS EXPERIENCE}

doi:10.1136/heartjnl-2012-301877b.65

S S Furniss, ${ }^{*}$ S J Podd, A Bandali, J Hunt, G Dhillon, A N Sulke. Eastbourne District General Hospital , East Sussex, UK

Introduction The practice of atrial fibrillation (AF) ablation is increasing exponentially. At present, these procedures are performed in a small number of surgical tertiary centres. In order to meet the growing demand it will be necessary to expand this service. We sought to assess patient satisfaction, procedural success and complication rates for AF ablation performed via a newly established district general hospital (DGH) service.

Methods We prospectively surveyed patients undergoing AF ablation, between November 2007 and November 2010, at Eastbourne DGH. Patient symptoms were assessed before and at least 12 months after AF ablation by telephone questionnaire, procedural satisfaction was also assessed. Procedural details (procedural time, fluoroscopy time, ablation technique) and complications were all accurately recorded for every case.

Results In 278 patients 314 procedures (average 1.13 procedures/pt) were performed (160 for persistent AF [PersAF], 134 for paroxysmal AF [PAF]). Mean age 63.8 10.7 years, $70 \%$ male. Of the PAF cases 75 procedures were performed with the PVAC (Medtronic, USA) and 56 with the Mesh (Bard, USA) catheter. The remaining three 